\title{
Histopathological Evaluation of Urethroplasty with Dorsal Buccal Mucosa: An Experimental Study in Rabbits
}

\author{
Geovanne F. Souza, Adriano A. Calado, Rosana Delcelo, Valdemar Ortiz, Antonio Macedo Jr. \\ Divisions of Urology (GFS, AAC, VO, AMJ) and Pathology (RD), Federal University of Sao Paulo, \\ UNIFESP, Sao Paulo, SP, Brazil
}

\begin{abstract}
Purpose: Buccal mucosa is a widely accepted tissue for urethroplasty. The exact healing and tissue integration process, mainly the histological characteristics of dorsal buccal mucosa graft urethroplasty when used dorsally to reconstruct the urethral plate has not previously been assessed, and thus we developed an experimental model to address this question. Materials and Methods: In 12 New Zealand rabbits (weight $2.5 \mathrm{~kg}$ ) we surgically created a dorsal penile urethral defect. A buccal mucosa graft was sutured to the corpora and tunica albuginea, and the ventral urethra anastomosed to this new urethral plate. The animals were divided in three groups and sacrificed 1, 3 and 6 weeks after surgery (groups 1, 2 and 3). A retrograde urethrogram was obtained at autopsy in the last group and the penis analyzed histologically with hematoxylin-eosin and Masson's staining.

Results: The urethrograms showed no evidence of fistula or stricture. In group 1 the histopathological analysis showed submucosal lymph-mononuclear inflammatory edema, numerous eosinophils and squamous epithelium integrated into the adjacent urothelium. In group 2 there was no evidence of an inflammatory response but rather complete subepithelial hyaline healing, which was more marked in group 3.

Conclusion: Healing of buccal mucosa grafts to reconstruct the urethral plate can be achieved by total integration of the squamous epithelium with the urothelium, maintaining the original histological properties of the graft with no fibrosis or retraction.
\end{abstract}

Key words: urethra; buccal mucosa; rabbits; experimental; surgery

Int Braz J Urol. 2008; 34: 345-54

\section{INTRODUCTION}

Urethral reconstruction under several pathologic conditions, such as strictures, traumatic defects, epispadias, and mainly in hypospadias, is one of the oldest problems in reconstructive surgery and one of the greatest surgical challenges for the surgeon. A variety of donor tissues have been used both experimentally and clinically for urethral repair, including free penile or preputial graft (1), hairless-skin grafts (2), bladder mucosal graft (3), buccal mucosal graft
(4), tunica vaginalis graft (5), peritoneal graft (6), intestinal submucosal graft (7) and more recently the tongue (8). Some of these methods have met with limited success and subsequently were abandoned.

Buccal mucosa grafting for urethroplasty of both urethral stricture and hypospadias repair has gained widespread acceptance during the past 10 years. With the initial description by Humby dating back to 1941, the method was reintroduced into the urologic literature in 1992 by Mainz et al. (4). Reported clinical results in literature have been extremely 
favorable both using the buccal mucosa as the ventral or dorsal component of the neourethra $(9,10)$.

Despite wide clinical use, little is known about the underlying mechanisms that incorporate the buccal mucosa graft into the urethral defect. A thorough understanding of this process could improve clinical outcome, which was achieved after defining the mechanisms of buccal mucosa grafting.

The aim of the present study was to investigate how healing progresses after dorsal buccal mucosa graft urethroplasty in a rabbit model, and the histopathological outcome of the procedure.

\section{MATERIAL AND METHODS}

Twelve New Zealand White rabbits aged approximately 6 weeks and weighing 2.0 to 2.5 $\mathrm{Kg}$ were acclimated in the Experimental Research Animal Surgery Department for one week before the study. The experimental protocol was reviewed and approved by the Local Animal Research Committee (approval no 1047/03).

The rabbits were anesthetized with intramuscular ketamine hydrochloride $(30 \mathrm{mg} / \mathrm{Kg})$ and intramuscular xylazine $(5 \mathrm{mg} / \mathrm{Kg})$ and received a preoperative dose of gentamicin $(1 \mathrm{mg} / \mathrm{Kg})$. After an adequate level of anesthesia was achieved, the penis was anesthetized with xylocaine and a $6 \mathrm{~F}$ urethral catheter was inserted. Under sterile conditions, the penis was released by dividing the perineal skin web between the ventral aspect of the penis and the anus. Each urethra was surgically exposed and operated under optical magnification (surgical microscope - 10x).

The urethra was carefully dissected and mobilized off the tunica albuginea. After exposing the urethra, a dorsal segment measuring $1.0 \times 1.0 \mathrm{~cm}$ was excised in all rabbits (urethral defect). A buccal mucosal graft was harvested from the cheek and tailored according to the area of the removed tissues. The graft was obtained by sharp dissection with fine scissors. The dissection was facilitated by prior submucosal injection of saline solution. The resulting wound was left open after careful coagulation of bleeding vessels.

The buccal mucosa graft was placed dorsally over the corpora cavernosa and tied with six inter- rupted polygalactin (Vicryl) 7-0 sutures. The mucosal margin of the urethral defect was sutured to the graft using 7-0 Vicryl sutures in a continuous fashion. The mucosal surface of the graft was always placed as the lumen of the reconstructed urethra. The skin was closed with a running 4-0 Vicryl stitch. Neither stent nor dressing was used. The operative technique is outlined in Figure-1.

The animals were recovered and returned to our chronic care facility. The animals were examined daily to monitor wound healing.

The experimental animals were divided into three equal groups and were sacrificed at 7 days, 3 weeks and 6 weeks after surgery, respectively. A retrograde urethrogram was taken at autopsy in the last group.

At the scheduled sampling time the animals were sacrificed with an overdose of Ketamine injection. The entire penis was examined and removed. The penises were fixed in 10\% formaldehyde and transverse sections cut to produce segments of $5 \mathrm{~mm}$ each, processed into paraffin blocks, and serially sectioned and stained with hematoxylin-eosin and Masson's trichrome. An experienced pathologist (RD) examined the specimens and evaluated the severity of acute and chronic inflammation, foreign body reaction, and scar formation. Masson's trichrome stain was used to localize collagen. With Masson's trichrome stain, the nuclei stained from deep mauve to black, cytoplasmic elements red and blue, muscle red and collagen-mucus green.

\section{RESULTS}

There were no deaths related to the procedure and all animals survived their intended survival period without evidence of infection, voiding difficulties, or fistula formation.

There were no difficulties associated with buccal mucosa harvesting and the macroscopic appearance of the operated penises was normal.

One week after surgery the buccal mucosa graft area had a proliferation reaction in all rabbits. There was no significant necrosis or erosion of any graft. A moderate infiltration of polymorphonuclear cells was observed, representing an acute inflammatory reaction (Figure-2). 


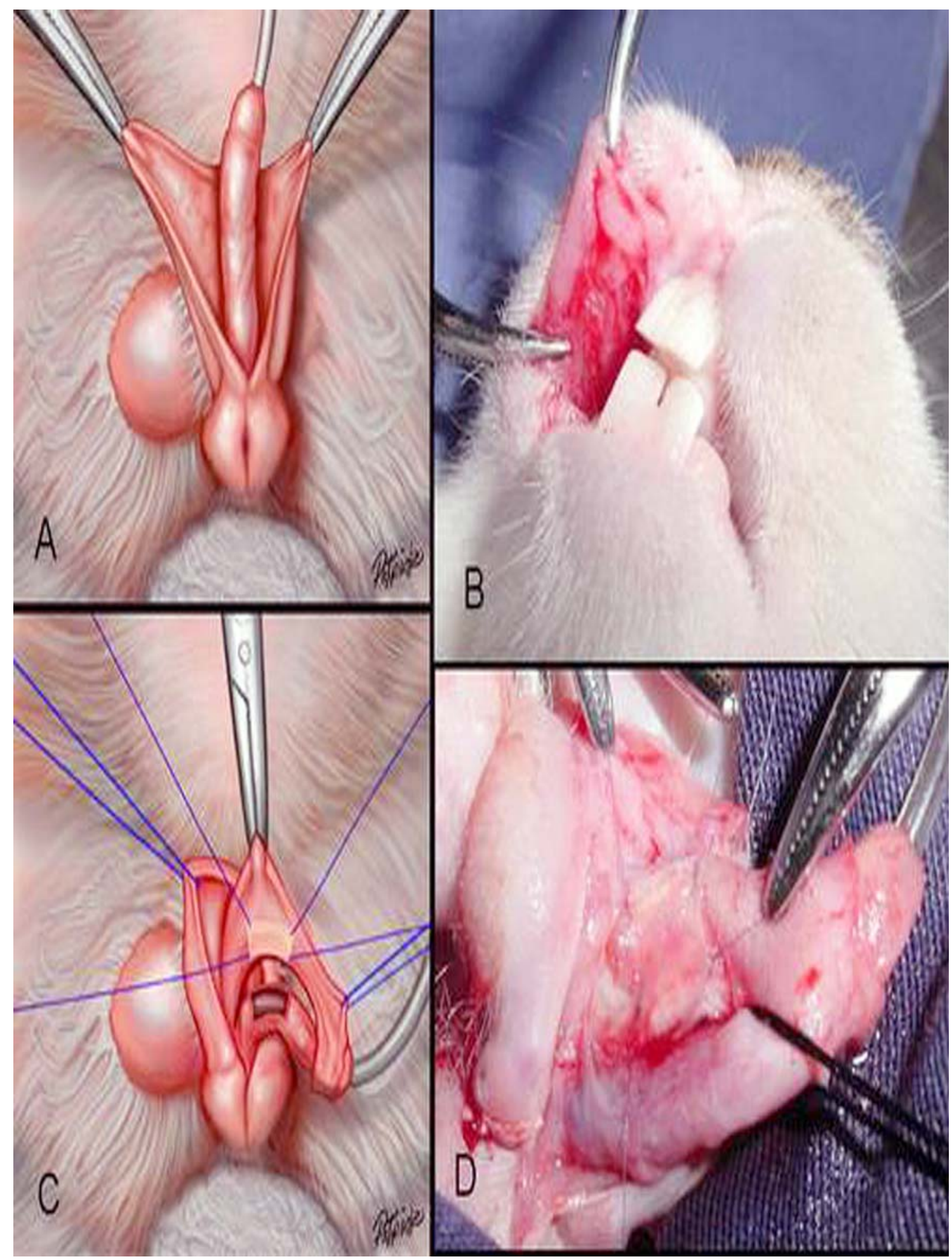

Figure 1 -Operative technique. A) The penis is degloved and the urethra is dissected and mobilized. B) Buccal mucosa graft was harvested from the cheek. C) and D) The buccal mucosa graft was placed dorsally over the corpora cavernosa and tied with six interrupted polygalactin (Vicryl) 7-0 sutures. 


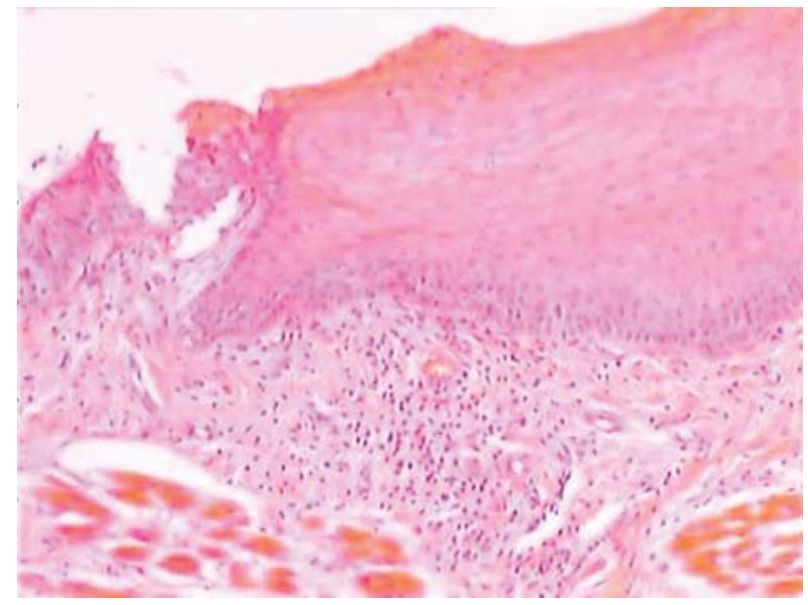

Figure 2 - The buccal mucosa graft one week after surgery. A moderate infiltration of polymorphonuclear cells was observed.

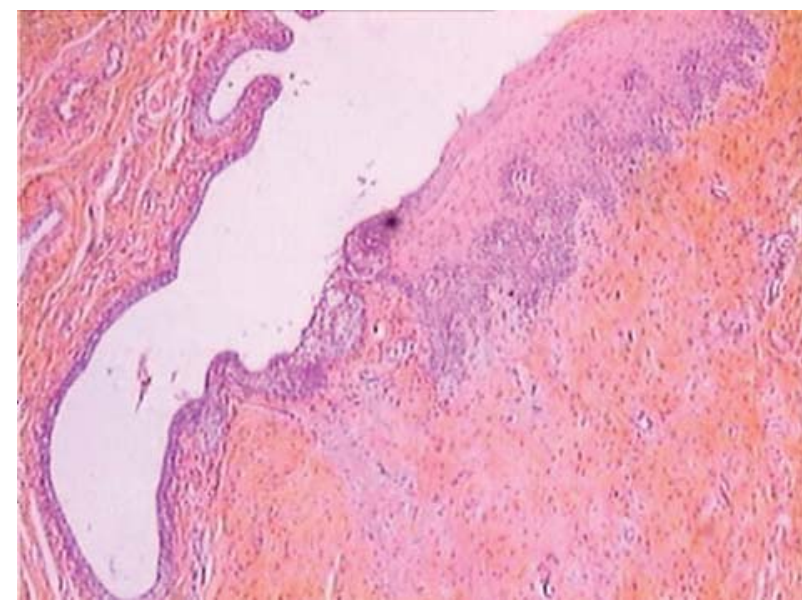

Figure 3 - The buccal mucosa graft three weeks after surgery. Extensive neovascularization was evident in the subepithelial layer with a streaming of fibroblasts toward the graft.

Three weeks after surgery extensive neovascularity was evident in the subepithelial layer with a streaming of fibroblasts toward the graft (Figure-3). Complete disappearance of the polymorphonuclear cells, representing resolution of the inflammatory reaction was evident by 6 weeks postoperatively. The histological appearance of the graft at postoperative week 6 is shown in Figure-4.

The typical squamous epithelium of buccal mucosa and minimal inflammatory cell infiltration in the subepithelial tissues were observed in all rabbits

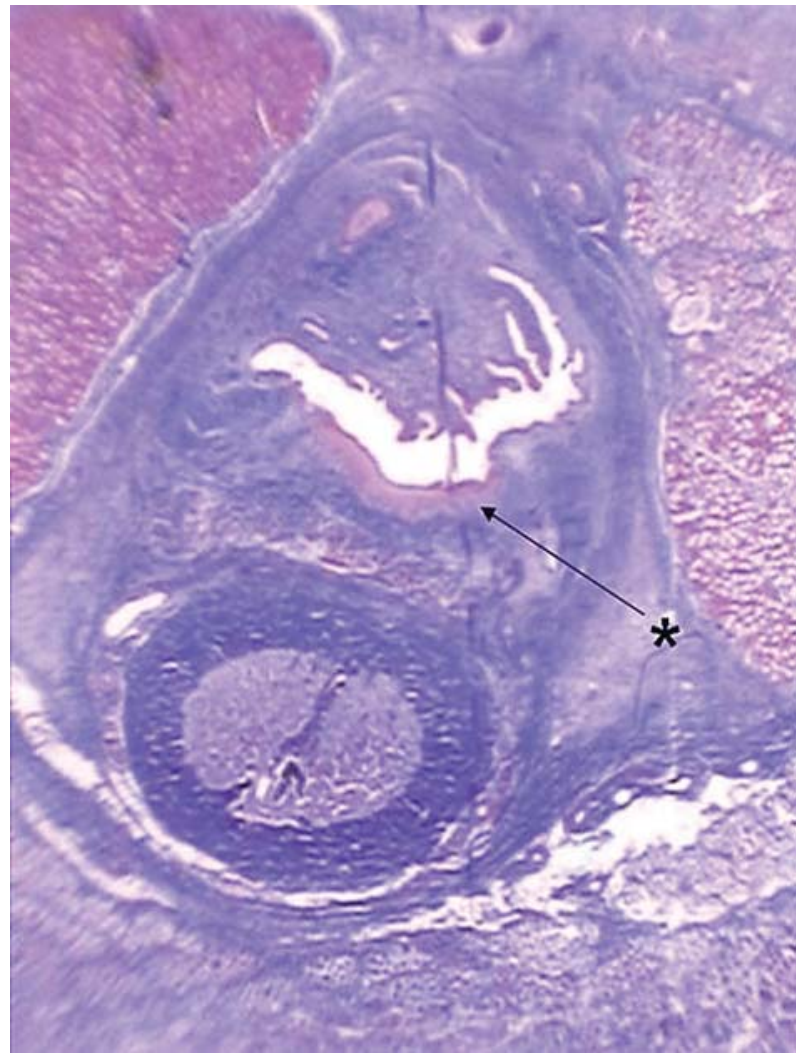

Figure 4-The histological appearance of the graft at postoperative week six.

at the grafted buccal mucosa six weeks after surgery. Minimal fibrosis was observed. Microscopically the junction of the graft and normal urethra was identifiable in all groups. Six weeks after surgery retrograde urethrograms confirmed the maintenance of a wide urethral caliber without any signs of stricture or extravasation (Figure-5).

\section{COMMENTS}

The choice of the substitute material for urethroplasty during hypospadias repair is the most important factor in determining the resulting complication rate for each surgical technique in urethral reconstruction; thus, a controversial debate is ongoing about the ideal material, especially in the repair of complex hypospadias. 


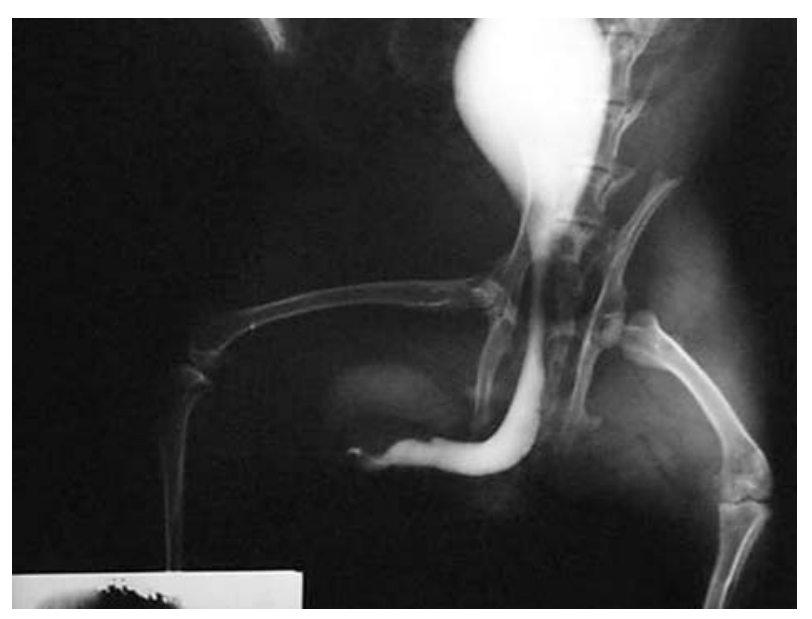

Figure 5 - Retrograde urethrogram six weeks after surgery confirmed the maintenance of a wide urethral caliber without any signs of stricture or extravasation.

Currently, buccal mucosa has become increasingly popular among pediatric urologists for urethral replacement during complex hypospadias repairs when local epithelial tissue is unavailable. Initial reports by Duckett et al.(11), Baskin and Duckett (12), and Burger et al. (4) have reported series with relatively low complication rates. Buccal mucosa seems to have distinct advantages over other materials due to its high degree of histological similarity to the normal urethra as revealed by morphology studies (13).

In contrast to bladder mucosa and penile skin, buccal mucosa comprises a thin submucosal and a thick epithelial layer. Whereas the thin submucosa may be important for fast and easy revascularization, stability of the material seems to be provided by the thick epithelial layer.

Additional immunohistochemistry studies have revealed a similar cytokeratin pattern between buccal mucosa and normal urethra as well as a parallel amount of immunoglobulin A in both tissues (13).

Although buccal mucosa grafts are performed for the surgical reconstruction of urethral problems, little is known about the mechanism by which engraftment occurs.

In the current study we developed a rabbit model to study the temporal healing process after a buccal mucosa dorsal graft urethroplasty. This rabbit model was used by our group in previous urethral reconstructive studies with success (14). In this model we used the tunica vaginalis graft placed dorsally anchored directly to the corpora as proposed by Barbagli et al.(15).

Barbagli et al. (15) argue that the dorsal location represents the best blood supply for graft take, prevents diverticulum formation, and is technically easier than a flap procedure. They also suggest that ventral placement of the graft leads to diverticulum formation and may impair the spongiosal blood supply.

In 2002, El-Sherbiny et al. (16) used an animal model of adult male mongrel dogs to compare the functional and pathological characteristics of three types of graft materials (buccal mucosa, bladder mucosa and free full-thickness skin) for urethroplasty. Buccal mucosa grafts were associated with the lowest rate of complications ( $12 \%$ ), followed by bladder mucosa $(37 \%)$ and free skin grafts $(62 \%)$. Filipas et al. (13) reported the result of histological and immunohistochemical pattern of full-skin and buccal mucosa grafts after exposure to urine in a pig model and indicated that the buccal mucosal graft showed significantly fewer adverse histopathological findings after long-term exposure to urine than the full-skin graft and is therefore a preferable material for urethral reconstruction.

It is widely accepted based on experimental experience that the thickness of the lamina propria, and especially the degree of native vascularity of the donor and recipient sites, influence the chances of graft take. Nevertheless, the viability of a graft depends on the neovascularization. In our study the buccal mucosa showed great formation of neovascularity three weeks after surgery.

To our knowledge the present experimental study is the first to describe the histological end aspect of the healing process of the buccal mucosa urethroplasty. We did not intend to define which physiological parameters or cytokeratins were involved but primarily to understand whether the buccal mucosa maintains its histological characteristics or undergoes metaplasia. In this study animals were sacrificed at 1,3 and 6 weeks postoperative, because this interval was considered to provide sufficient time for wound healing. 
The experimental study here presented reinforces the role of dorsal buccal mucosa as an excellent tissue source for urethral reconstruction. Despite widespread use in urethral stricture, it has only recently been incorporated to hypospadias repair in a two-step approach basis $(17,18)$. Snodgrass et al. (18) reported outcomes from staged buccal graft urethroplasty after failed hypospadias surgery. In this study 25 patients underwent stage 1 repair following an average of 4.4 prior hypospadias surgeries and 20 patients underwent stage 2 . There were no cases of meatal stenosis, neourethral stricture or diverticulum. The authors concluded that staged buccal graft reoperation reliably creates a well vascularized substitute urethral plate for tubularization with low complication rates and good cosmetic outcomes.

We have used the dorsal buccal mucosal graft as a way to reconstruct the urethral plate after urethral plate section to straighten the penis in complex primary hypospadia forms (19). This step restores the continuity of the urethral plate and allows the use of a preputial flap that can be anchored onlay to the buccal mucosa concomitantly. We have previously presented the technique and the outcome of initial results and outcome has proved to be very favorable (19).

The patency of the urethra in radiological studies and the fine histopathological integration of dorsal buccal mucosa to the native urethral mucosa as shown here in a single procedure, support our concept of dorsal grafting plus onlay ventral flap as a useful and viable strategy for one-step urethral reconstruction in almost every complex primary hypospadia patient.

Interestingly the same study performed using the tunica vaginalis as the dorsal part of the urethra showed that this tissue, being different from the buccal mucosa, changes its histological properties and resembles the urethral epithelium (14). Nevertheless, the concept of dorsal grafting plus onlay flap in urethroplasty seems to function independently from the dorsal component (buccal mucosa or tunica vaginalis). We stress that the availability of long term follow-up studies using buccal mucosa in urethral reconstruction justifies our present preference for its use as first choice tissue in hypospadia repair (the three-in-one technique). We hypothesized that tunica vaginalis could have the same place as an alternative source of tissue for dorsal graft urethroplasty. However, further clinical series with long term follow-up would be required to confirm this theory. The authors also agree that "the three-in-one concept" in the clinical setting deserves long term approval although it could be regarded as a valuable option.

We also accept that the results found in the present study performed in an untouched urethra may vary when treating a recurrent failed urethral repair and only a specific study under similar conditions could provide a definite conclusion regarding urethral substitution surgery.

\section{CONCLUSION}

A urethroplasty with dorsal buccal mucosa in rabbits showed total integration to the adjacent epithelium, maintaining their histological characteristics, without occurrence of fibrosis, retraction or necrosis.

\section{CONFLICT OF INTEREST}

None declared.

\section{REFERENCES}

1. Stock JA, Cortez J, Scherz HC, Kaplan GW: The management of proximal hypospadias using a 1-stage hypospadias repair with a preputial free graft for neourethral construction and a preputial pedicle flap for ventral skin coverage. J Urol. 1994; 152: 2335-7.

2. Devine CJ Jr, Horton CE: A one stage hypospadias repair. J Urol. 196; 85: 166-72.

3. Memmelaar J: Use of bladder mucosa in a one-stage repair of hypospadias. J Urol. 1947; 58: 68-72.

4. Bürger RA, Müller SC, el-Damanhoury H, Tschakaloff A, Riedmiller H, Hohenfellner R: The buccal mucosal graft for urethral reconstruction: a preliminary report. J Urol. 1992; 147: 662-4.

5. Snow BW, Cartwright PC: Tunica vaginalis urethroplasty. Urology. 1992; 40: 442-5.

6. Shaul DB, Xie HW, Diaz JF, Mahnovski V, Hardy BE: Use of tubularized peritoneal free grafts as urethral substitutes in the rabbit. J Pediatr Surg. 1996; 31: 225-8. 
7. Kropp BP, Ludlow JK, Spicer D, Rippy MK, Badylak $\mathrm{SF}$, Adams MC, et al.: Rabbit urethral regeneration using small intestinal submucosa onlay grafts. Urology. 1998; 52: 138-42.

8. Simonato A, Gregori A, Lissiani A, Galli S, Ottaviani F, Rossi R, et al.: The tongue as an alternative donor site for graft urethroplasty: a pilot study. J Urol. 2006; 175: 589-92.

9. Bhargava S, Chapple CR: Buccal mucosal urethroplasty: is it the new gold standard? BJU Int. 2004; 93: 1191-3.

10. Dubey D, Kumar A, Mandhani A, Srivastava A, Kapoor R, Bhandari M: Buccal mucosal urethroplasty: a versatile technique for all urethral segments. BJU Int. 2005; 95: 625-9.

11. Duckett JW, Coplen D, Ewalt D, Baskin LS: Buccal mucosal urethral replacement. J Urol. 1995; 153: 16603.

12. Baskin LS, Duckett JW: Buccal mucosa grafts in hypospadias surgery. Br J Urol. 1995; 76 (Suppl 3): 23-30.

13. Filipas D, Fisch M, Fichtner J, Fitzpatrick J, Berg K, Störkel S, et al.: The histology and immunohistochem-

\section{Correspondence address:}

Dr. Adriano Almeida Calado

Rua Afonso de Albuquerque Melo, 420/1202

Recife, PE, 52060-450, Brazil

E-mail: caladourologia@yahoo.com.br

\section{EDITORIAL COMMENT}

After the original publication by Orandi in June 1968 at the 24th Annual Meeting of the British Association of Urological Surgeons in Cardiff (1), the one-stage flap urethroplasty, based on Orandi's original suggestions, was popularized by Quartey, McAninch and Jordan (2-4). In 1994, Snodgrass, was the first to describe the tabularized, incised plate istry of free buccal mucosa and full-skin grafts after exposure to urine. BJU Int. 1999; 84: 108-11.

14. Calado AA, Macedo A Jr, Delcelo R, de Figueiredo LF, Ortiz V, Srougi M: The tunica vaginalis dorsal graft urethroplasty: experimental study in rabbits. J Urol. 2005; 174: 765-70.

15. Barbagli G, Selli C, di Cello V, Mottola A: A one-stage dorsal free-graft urethroplasty for bulbar urethral strictures. Br J Urol. 1996; 78: 929-32.

16. El-Sherbiny MT, Abol-Enein H, Dawaba MS, Ghoneim MA: Treatment of urethral defects: skin, buccal or bladder mucosa, tube or patch? An experimental study in dogs. J Urol. 2002; 167: 2225-8.

17. Manzoni G, Bracka A, Palminteri E, Marrocco G: Hypospadias surgery: when, what and by whom? BJU Int. 2004; 94: 1188-95.

18. Snodgrass W, Elmore J: Initial experience with staged buccal graft (Bracka) hypospadias reoperations. J Urol. 2004; 172: 1720-4; discussion 1724.

19. Macedo A Jr, Srougi M: Onlay urethroplasty after sectioning of the urethral plate: early clinical experience with a new approach - the 'three-in-one' technique. BJU Int. 2004; 93: 1107-9.

Accepted after revision: April 26, 2008

urethroplasty for distal hypospadias repair (5). After 5 years due to the description of these techniques, Hayes and Malone suggested laying an oral mucosal graft into Snodgrass' midline incision of the urethral plate in patients with failed hypospadias repair (6). In this past decade, the interest in buccal mucosa as a substitute material in the reconstruction of the penile 
urethra has been attracting the attention of most of reconstructive surgeons. Recently, Barbagli et al. provided a retrospective evaluation of the outcome in patients who underwent one-stage penile flap or graft urethroplasty (7). These authors found that the use of grafts for one-stage penile urethroplasty showed a higher success rate $(80.0 \%)$ compared to flaps $(66.7 \%)$. The difference in the success rate between oral mucosal grafts and skin grafts was not clinically significant.

Souza and co-workers should be praised for their study as it introduces readers to an aspect of "urethral basic science" and provides some answers to the knowledge gap. They performed a histopathological assessment of the exact healing process of buccal mucosa graft, when it was used dorsally to reconstruct the urethral plate in vivo animal model of penile stricture. After surgically creating a dorsal penile urethral defect, a buccal mucosa graft was sutured to the corpora and tunica albuginea. Animals were stratified into three different groups according to timing of histopathological analysis. After one week histopathological analysis showed submucosal lymph-mononuclear inflammatory edema, numerous eosinophils and squamous epithelium integrated into the adjacent urothelium (Group-1). After 3 weeks there was no evidence of an inflammatory response but complete subepithelial hyaline healing (Group-2), which was more marked after 6 weeks (Group-3). The authors concluded that the healing process of buccal mucosa grafts, used for reconstructing the urethral plate is by total integration of the squamous epithelium with the urothelium, maintaining the original histological properties of the graft with no fibrosis or retraction.

Currently, oral mucosa seems to be unsurpassed as donor substitute material in adult anterior urethroplasty, however pediatric and general urologists who are involved in the reconstruction of urethra are facing new challenges. What is the ideal harvest site? The most common harvest sites for oral mucosa are the lower lip and the cheeks. Simonato et al. and Barbagli et al. recently reported the tongue as an alternative donor site in graft urethroplasty $(8,9)$. Furthermore what could be the role of tissue engineering? All these issues will be addressed in the near future.

\section{REFERENCES}

1. Orandi A: One-stage urethroplasty. Br J Urol. 1968; 40: 717-9.

2. Quartey JK: One-stage penile/preputial cutaneous island flap urethroplasty for urethral stricture: a preliminary report. J Urol. 1983; 129: 284-7.

3. McAninch JW: Reconstruction of extensive urethral strictures: circular fasciocutaneous penile flap. J Urol. 1993; 149: 488-91.

4. Jordan GH, Stack RS: General concepts concerning the use of genital skin islands for anterior urethral reconstruction. Atlas Urol Clin N Am. 1997; 5: 23-44.

5. Snodgrass W: Tubularized, incised plate urethroplasty for distal hypospadias. J Urol. 1994; 151: 464-5.

6. Hayes MC, Malone PS: The use of a dorsal buccal mucosal graft with urethral plate incision (Snodgrass) for hypospadias salvage. BJU Int. 1999; 83: 508-9.

7. Barbagli G, Morgia G, Lazzeri M: Retrospective outcome analysis of one-stage penile urethroplasty using flap or graft in a homogeneous series of 63 patients. Br J Urol. 2008; in press.

8. Simonato A, Gregori A, Ambruosi C, Venzano F, Varca V, Romagnoli A, et al.: Mucosal graft urethroplasty for anterior urethral reconstruction. Eur Urol. 2008 Jan 16 [Epub ahead of print].

9. Barbagli G, De Angelis M, Romano G, Ciabatti PG, Lazzeri M: The use of lingual mucosal graft in adult anterior urethroplasty: surgical steps and short-term outcome. Eur Urol. 2007 Dec 18 [Epub ahead of print].

Dr. Massimo Lazzeri

Department of Urology, Santa Chiara Hospital Florence, Italy

E-mail:lazzeri.m@tiscali.it

Dr. Guido Barbagli Center for Reconstructive Urethral Surgery Arezzo, Italy 


\section{EDITORIAL COMMENT}

For hypospadias repair more than 300 techniques and their modification have already been published (1). Buccal mucosa can be used in primary reconstruction, as well as after failed reconstruction if no other material is available. Initial animal experience in dogs demonstrated that this technique is feasible. Buccal mucosa has been used at our institution for urethral reconstruction since 1990 (2). Placing the Buccal mucosa graft on the dorsal site was popularized by Barbagli et al. in 1998 (3).

Souza and colleagues from Brazil investigated the use of buccal mucosa in a rabbit model as a dorsal onlay in primary hypospadias repair. They divided the 12 rabbits into 3 groups according to scarification time for histopathological investigation. They observed acute inflammatory reaction after 7 days, a good neo-vascularization after three weeks and some resolution of the inflammatory reaction with minimal fibrosis after 6 weeks.

Although each group consists of only a few animals $(n=4)$ and the results are confined to a description of the histopathological findings with no quantification, this study demonstrates that buccal mucosa causes no severe reaction if used for urethral reconstruction.

However, this experiment has its limitations. The authors used buccal mucosa in healthy tissue, and the animals had no previous surgery. It would be interesting to see what would happen if buccal mucosa was used for secondary reconstruction. The authors completed their experiment after 6 weeks. It would have been of some interest to see what would happen in the long run. Is there more fibrosis? Does the urothelium replace the buccal mucosa? Does the buccal mucosa undergo changes? These questions should be addressed in further long-term studies. This study is one of the first to investigate histopathological findings after urethral repair using buccal mucosa. More studies should be performed, in particular from the aspect of tissue engineering using buccal mucosa.

\section{REFERENCES}

1. Schröder A, Stein R, Melchior S, Fisch M, Riedmiller H, Thüroff JW: Hypospadie. Urologe A. 2006; 45(Suppl 4): 204-208.

2. Bürger RA, Müller SC, el-Damanhoury H, Tschakaloff A, Riedmiller H, Hohenfellner R: The buccal mucosal graft for urethral reconstruction: a preliminary report. J Urol. 1992; 147: 662-4.

3. Barbagli G, Palminteri E, Rizzo M: Dorsal onlay graft urethroplasty using penile skin or buccal mucosa in adult bulbourethral strictures. J Urol. 1998; 160: 13079.

\author{
Dr. Raimund Stein \\ Department of Urology \\ University of Mainz \\ Mainz, Germany \\ E-mail:stein@urologie.klinik.uni-mainz.de
}




\section{EDITORIAL COMMENT}

We congratulate the authors, which developed an elegant experimental model in the rabbit in order to evaluate the healing progress and the histopathological outcome of the dorsal buccal mucosa graft urethroplasty. The results of the study indicate that the buccal mucosa shows total integration to the adjacent epithelium, maintaining the histological characteristics without occurrence of fibrosis, retraction or necrosis.

In February 2006 we described the results of a pilot study on the use of the tongue (lingual mucosa graft - LMG) as an alternative donor site for graft urethroplasty with good functional and aesthetic results (1). We performed a urethral biopsy of LMG after 3 months which revealed absent pathological alterations in the nonkeratinizing, stratified lingual epithelium (1).

After this preliminary experience, our group (2) and other authors $(3,4)$ confirmed that LMG is an excellent graft material with the advantage of potential minor donor site complications. A specific study on the donor site morbidity associated to a LMG provided further evidence that LMG may be harvested with only temporary donor site discomfort and without long term complications, confirming that the tolerability of the harvesting procedure is very high with minor risks of donor site complications (5). Minor donor site morbidity was also obtained by otorhinolaryngologists (6), which had an awaked interest in using LMG after our pilot study (1) to reconstruct and restore epithelial continuity of buccal/lip mucosal defects after tumour resection.

It would be very interesting if the authors could apply their experimental rabbit model, if tech- nically possible, to evaluate if total integration to the adjacent epithelium with maintenance of the histological characteristics occur when a LMG is used for urethroplasty. In this way we may have a comparison of the lingual and buccal mucosa grafts.

\section{REFERENCES}

1. Simonato A, Gregori A, Lissiani A, Galli S, Ottaviani F, Rossi R, et al.: The tongue as an alternative donor site for graft urethroplasty: a pilot study. J Urol. 2006; 175: 589-92.

2. Simonato A, Gregori A, Ambruosi C, Venzano F, Varca V, Romagnoli A, et al.: Lingual Mucosal Graft Urethroplasty for Anterior Urethral Reconstruction. Eur Urol. 2008; Jan 16. [Epub ahead of print]

3. Barbagli G, De Angelis M, Romano G, Ciabatti PG, Lazzeri M: The Use of Lingual Mucosal Graft in Adult Anterior Urethroplasty: Surgical Steps and Short-Term Outcome. Eur Urol. 2007; Dec 18. [Epub ahead of print]

4. Kumar A, Das SK, Sharma GK, Pandey AK, Trivedi S, Dwivedi US, et al.: Lingual mucosal graft substitution urethroplasty for anterior urethral strictures: our technique of graft harvesting. World J Urol. 2008; Apr 19. [Epub ahead of print]

5. Kumar A, Goyal NK, Das SK, Trivedi S, Dwivedi US, Singh PB: Oral complications after lingual mucosal graft harvest for urethroplasty. ANZ J Surg. 2007; 77: 970-3.

6. Lai CC, Su CY: Free mucosa graft from the lateral tongue for reconstruction of intraoral buccal/lip mucosal defects after tumor resection. Laryngoscope. 2007; 117: 1368-72.

Dr. Alchiede Simonato Dr. Andrea Gregori Clinica Urologica "L.Giuliani" University of Genoa Azienda Ospedaliera Universitaria San Martino Genova, Italy E-mail: alchiede.simonato@unige.it 